\title{
A Autoestima e a Insatisfação Corporal estão Associadas aos Motivos para a Prática de Hidroginástica em Mulheres Idosas?
}

\section{Are Self-Esteem and Body Dissatisfaction Associated with the Reasons for the Practice of Water Aerobics in Elderly Women?}

\section{RESUMO}

Objetivo: Analisar a associação da autoestima e a insatisfação corporal com os motivos para a prática de hidroginástica em idosas. Metodologia: Participaram 70 idosas praticantes de hidroginástica em academias na Cidade de Maringá-PR, há no mínimo três meses. Como instrumentos foram utilizados um questionário sociodemográfico, a Escala de autoestima de Rosemberg, a Escala de silhuetas e o Exercise motivation inventory (EMI-2). A análise dos dados foi conduzida por meio dos testes de Kolmogorov-Smirnov, Mann-Whitney, correlação de Spearman e Path Analysis $(p<0,05)$. Resultados: Os resultados evidenciaram que as idosas com presença de distúrbio de autoestima são mais motivadas para competir e serem reconhecidas socialmente. As idosas satisfeitas com o próprio corpo são mais motivadas a praticar a hidroginástica para a prevenção de doenças. Ocorreu correlação significativa $(p<0,05)$ do fator de condição física com a autoestima $(r=$ $0,28)$ e do fator de diversão com a autoestima $(r=0,32)$. O modelo de Path analysis revelou que a autoestima apresentou efeito moderado sobre os fatores de condição física $(\beta=0,22)$ e diversão $(\beta=0,24)$. Conclusão: Concluiu-se que a autoestima e a insatisfação corporal são fatores intervenientes na motivação de idosas para a prática de atividade física. Ressalta-se que a autoestima pode favorecer a busca pela atividade física para melhorar a saúde física e para a diversão.

\section{DESCRITORES}

Atividade Motora. Exercício. Envelhecimento. Psicologia do Esporte.

\begin{abstract}
Objective: This cross-sectional study aimed to analyze the association of self-esteem and body dissatisfaction with the reasons for the practice of water aerobics for elderly women. Methodology: 70 elderly women who practiced water aerobics in gyms in the city of Maringá-PR for at least three months participated in the study. As instruments, a sociodemographic questionnaire, the Rosemberg Self-Esteem Scale, the Silhouettes Scale, and the Exercise Motivation Inventory (EMI-2) were used. Data analysis was performed using the Kolmogorov-Smirnov, Mann-Whitney tests, Spearman correlation and Path Analysis $(p<0.05)$. Results: The results showed that elderly women with self-esteem disorders are more motivated to compete and be socially recognized. Elderly women satisfied with their own bodies are more motivated to practice water aerobics for disease prevention. There was a significant correlation $(p<0.05)$ between the physical condition factor and self-esteem $(r=0.28)$ and the fun factor with selfesteem ( $r=0.32$ ). The Path Analysis model revealed self-esteem and had a moderate effect on the factors of physical condition $(\beta=0.22)$ and fun $(\beta=0.24)$. Conclusion: It was concluded that self-esteem and body dissatisfaction are intervening factors in the motivation of elderly women to practice physical activity. It is noteworthy that self-esteem can favor the search for physical activity to improve physical health and for fun.
\end{abstract}

\section{DESCRIPTORS}

Motor Activity. Exercise. Aging. Sport Psychology.

${ }_{1}^{1}$ Doutor em Gerontologia, Departamento de Educação Física, Centro Universitário Metropolitano de Maringá, Maringá, PR, Brasil.

${ }^{2}$ Graduada em Educação Física, Departamento de Educação Física, Centro Universitário Metropolitano de Maringá, Maringá, PR, Brasil.

${ }^{3}$ Mestrando em Educação Física, Programa de Pós-Graduação em Educação Física, Universidade Federal do Vale do São Francisco, Petrolina, PE, Brasil.

${ }^{4}$ Doutorando em Ciências da Reabilitação, Departamento de Fonoaudiologia, Fisioterapia e Terapia Ocupacional, Faculdade de Medicina da Universidade de São Paulo, São Paulo, SP, Brasil.

${ }^{5}$ Doutor em Educação Física, Programa de Pós-Graduação em Educação Física, Universidade Federal do Vale do São Francisco, Petrolina, PE, Brasil. 
$\mathrm{A}$ população mundial vem envelhecendo constantemente, no Brasil, isso não diferente, estimando-se que o número de idosos extrapole os 20 milhões. O envelhecimento se compreende como um processo progressivo, seguido por alterações físicas, sociais e psicológicas ${ }^{1}$.

No processo de envelhecimento, um fator que interfere na busca pela atividade física em idosos é a autoimagem e a autoestima, que são variáveis psicológicas interligadas ${ }^{2}$. Quando a autoestima é alta, decorre de experiências positivas com a vida. Por outro lado, quando a autoestima é baixa, resulta de fatores negativos ${ }^{2}$. Copatti et al. ${ }^{3}$ asseguram que a autoimagem está sempre em mudança, decorrente das experiencias adquiridas na vida cotidiana, ocupacional e de lazer.

Outro fator que chama atenção para não adesão ao exercício físico é a insatisfação corporal. Estudos recentes estão identificando um aumento na prevalência de idosos com insatisfação ao seu próprio corpo, principalmente em mulheres ${ }^{4-5}$. As mudanças fisiológicas no envelhecimento afetam de forma direta a insatisfação corporal no sexo feminino, bem como, o estado nutricional em idosas 5 .

Embora se saiba que existem inúmeras barreiras para o idoso não se inserir num programa de exercício físico, pode-se apontar como a principal barreira para essa não adesão, a motivação. Biedenweg et al. ${ }^{6}$ asseguram que os idosos não se motivam para iniciar ou permanecer em um programa de exercício físico. Os autores ainda reforçam que a motivação tem como característica uma ação intencional, direcionada para uma meta e regulada por fatores ambientais e pessoais.
Uma das principais abordagens para o estudo da motivação tem sido a teoria da autodeterminação (TAD), que tem o propósito de interpretar a personalidade da motivação humana, bem como, a motivação intrínseca e extrínseca, analisando todos os aspectos favoráveis ou não para que a motivação possa ocorrer. Na prática do exercício físico, a TAD pode ser aplicada em distintos grupos populacionais e em diferentes contextos. Nessa perspectiva, investigações vêm trazendo resultados com o intuito de assimilar melhor os aspectos motivacionais dos idosos, na aderência a um programa de exercício físico ${ }^{7}$. Nesse sentido, existem inúmeros benefícios da atividade física durante o processo de envelhecimento. Estudo recente aponta a importância da prática de exercício físico na melhoria dos parâmetros físicos, fisiológicos e psicológicos ${ }^{8}$.

Com isso, uma melhor compreensão das experiências de idosos, crenças e atitudes em relação à pratica de exercícios físicos, ajuda a traduzir as fortes evidências dos benefícios da pratica de exercícios físicos para essa população. Diante disso, é importante estudar a prática de exercício em idosos, assim como a motivação para a prática do mesmo e se a autoestima e insatisfação corporal dessa população estão associadas aos motivos para a prática de exercício. Diante disso, o objetivo do presente estudo foi analisar a associação da autoestima e da insatisfação corporal com os motivos para a prática de hidroginástica de idosas.

\section{METODOLOGIA}

O processo de amostragem foi por 
conveniência, tendo a amostra sido composta por 70 idosas (60 anos ou mais), praticantes de hidroginástica, há no mínimo três meses, nas academias selecionadas, localizadas no Município de Maringá, Paraná. Foram excluídas as idosas com déficits auditivos perceptíveis e possíveis déficits cognitivos avaliados pelo $\mathrm{MEEM}^{9-10}$.

\section{Instrumentos}

Foi utilizado um questionário estruturado pelos autores, para avaliar o perfil sociodemográfico, de saúde e da prática de hidroginástica. $\mathrm{O}$ mesmo foi composto pelas seguintes questões: idade, faixa etária (60 a 69 anos; 70 a 79 anos; 80 a 89 anos; 90 anos ou mais), estado conjugal (com companheiro; sem companheiro), escolaridade (não estudou; fundamental incompleto; fundamental completo; médio completo; superior), renda mensal em salário (s) mínimo (s) (SM) (1 a $2 \mathrm{SM}$; 2,1 a $3 \mathrm{SM}$; mais de $3 \mathrm{SM}$ ), aposentadoria (sim; não), percepção de saúde (boa; regular; ruim), percepção de saúde atual comparada à outra pessoa da mesma idade (pior; igual; melhor), uso de medicamentos (sim; não), histórico de quedas e quase quedas no último semestre (sim; não), frequência da prática de hidroginástica (2x; $3 x$; $4 x$ ou mais), tempo de prática de hidroginástica ( 3 meses a 1 ano; 1,1, a 3 anos; mais de 3 anos).

A escala de autoestima de Rosemberg foi utilizada para avaliar a autoestima das idosas. É uma escala likert, contendo 10 itens, com a sua pontuação variando de 1 a 4 pontos, sendo "1" ponto "discorda totalmente" e a pontuação de "4" pontos "concorda totalmente". Quanto maior a pontuação final, maior a autoestima ${ }^{11}$.

A percepção da imagem corporal considerada real e ideal foi autorreferida por meio da escala de nove silhuetas, proposta por Stunkard et al. ${ }^{12}$, que classifica desde a magreza (silhueta 1) até a obesidade severa (silhueta 9). Para avaliação da percepção da imagem corporal, obteve-se a diferença entre a aparência corporal real e a aparência corporal ideal. Se a variação fosse igual a zero, classificava-se a idosa como satisfeita; e se diferente de zero, classificava-se como insatisfeita. Caso a diferença fosse positiva, considerou-se insatisfação pelo excesso de peso.

Para avaliar a motivação, foi aplicado o questionário Exercise Motivation Inventory (EMI-2). Segundo Bavoso ${ }^{11}$, este instrumento apresenta uma pontuação de "1" indicando "nada importante", até a pontuação "5", indicando "muito importante", respondendo questões que abrangem fatores motivacionais intrínsecos e extrínsecos, sobre a sua prática de exercício físico.

\section{Procedimentos}

Estudo transversal, aprovado pelo Comitê de Ética em Pesquisa do Centro Universitário de Maringá, por meio do Parecer $\mathrm{n}^{\circ}$. 1.745.616/2016, no qual a coleta de dados foi realizada em nove academias que ofereciam a modalidade de hidroginástica no município. Estas compõem, com exceção de uma, todos os locais no município que ofereçam essa modalidade.

As idosas foram abordadas antes ou após as aulas e, aquelas que aceitarem 
participar da pesquisa, assinarão o Termo de consentimento livre e esclarecido (TCLE). Optou-se pela forma de entrevista na coleta dos dados, a fim de evitar possíveis erros de leitura pelas idosas.

\section{Análise dos dados}

A análise dos dados foi realizada por meio do software SPSS 22.0, mediante uma abordagem de estatística descritiva e inferencial. Foi utilizado a frequência e o percentual, como medidas descritivas para as variáveis categóricas. Para as variáveis numéricas, inicialmente, foi verificada a normalidade dos dados por meio do teste Kolmogorov-Smirnov. A comparação dos fatores de motivação de acordo com o distúrbio de autoestima e a presença de insatisfação corporal foi realizada por meio do teste "U" de Mann-Whitney. A correlação entre a motivação, a autoestima e a insatisfação corporal foi verificada por meio do coeficiente de Spearman. Foi adotada a significância de $p<0,05$.

Para verificar o percentual de variância explicada dos motivos para a prática de hidroginástica pela autoestima e pela insatisfação corporal, foram conduzidos modelos de Path Analysis, com as variáveis que obtiveram correlação significativa $(p<0,05)$. A existência de outliers foi avaliada pela distância quadrada de Mahalanobis $\left(D M^{2}\right)$ e a normalidade univariada das variáveis foi avaliada pelos coeficientes de assimetria $(|S k|<3)$ e curtose $(I K u K<10)$ uni e multivariada. Como os dados não apresentaram distribuição normal, utilizou-se a técnica de Bootstrap de Bollen-Stine para corrigir o valor dos coeficientes estimados pelo método da máxima verossimilhança (Marôco, 2010), implementado no software AMOS versão 22.0. Para verificar a adequação da amostra para a análise proposta, aplicamos a técnica de Bootstrapping. Não foram observados valores de $D M^{2}$ indicadores da existência de outliers, nem correlações suficientemente fortes entre as variáveis que indicaram multicolinearidade (Variance Inflation Factors $<5,0)$. Partindo das recomendações de Kline (2012), a interpretação dos coeficientes de regressão teve como referência: pouco efeito para coeficientes $<0,20$, médio efeito para coeficientes até 0,49 e forte efeito para coeficientes $>0,50$.

\section{RESULTADOS}

Das 70 idosas participantes da pesquisa, observou-se a frequência de mulheres sem companheiro $(58,6 \%)$, com idade entre 60 e 69 anos (54,3\%), renda mensal de um a dois salários mínimos (65,4\%), da cor branca $(84,3 \%)$, aposentadas $(71,4 \%)$ e com ensino médio/superior completo $(41,4 \%)$. Verificou-se que a maioria das idosas possui percepção de saúde boa (54,3\%), toma mais de dois medicamentos regularmente $(57,1 \%)$ e não tinham histórico de quedas $(78,6 \%)$ nos últimos seis meses. Além disso, destaca-se que $67,1 \%$ das idosas praticavam a hidroginástica há mais de três anos e 62,9\% praticam a modalidade até duas vezes por semana.

Conforme os resultados da Tabela 1 , os principais motivos que levam as idosas a praticar a hidroginástica são: prevenção de doenças $(M d=5,0)$, afiliação $(M d=5,0)$, 
controle do estrese $(\mathrm{Md}=4,8)$ e diversão ( $M d$ $=4,7)$. Percebe-se também que as idosas apresentaram satisfatório nível de autoestima $(M d=34,0)$ e leve insatisfação pela magreza $(\mathrm{Md}=-1,0)$.

Ao comparar os fatores de motivação para a prática de hidroginástica das idosas em função do distúrbio de autoestima (Tabela 2), verificou-se diferença significativa apenas nos fatores de competição $(p=$ $0,037)$ e reconhecimento social ( $p=0,048)$, evidenciando que as idosas com presença de distúrbio de autoestima são mais motivadas para a prática da hidroginástica para competir e para serem reconhecidas socialmente do que as idosas com ausência de distúrbio de autoestima.

Verificou-se diferença significativa apenas no fator de motivação de prevenção de doenças ( $p=0,005)$ em função da insatisfação corporal (Tabela 3 ), indicando que as idosas satisfeitas com o próprio corpo ( $M d=5,00$ ) são mais motivadas a praticar a hidroginástica para a prevenção de doenças do que as idosas insatisfeitas com o próprio corpo (Md $=4,50)$. No entanto, ressalta-se que as idosas de ambos os grupos apresentaram alto escore neste fator de motivação. Não foi encontrada diferença significativa $(p>0,05)$ nos demais fatores de motivação.

$\mathrm{Na}$ análise da correlação entre a motivação para a prática da hidroginástica, a autoestima e a insatisfação corporal das idosas (Tabela 4), foi encontrada correlação significativa $(p<0,05)$ somente do fator de condição física com a autoestima $(r=0,28)$ e do fator de diversão com a autoestima $(r=0,32)$. Este achado indica que existe uma associação diretamente proporcional da procura pelo exercício para melhorar a condição física e para se divertir com a autoestima.

Para verificar o percentual de variância explicada dos motivos para a prática de hidroginástica pela autoestima e insatisfação corporal das idosas (Figura 1), após a análise da correlação, foi conduzido um modelo de Path Analysis entre as variáveis que apresentaram

Tabela 1. Estatística descritiva da motivação, autoestima e insatisfação corporal das idosas praticantes de hidroginástica

\begin{tabular}{lr}
\multicolumn{1}{c}{ Variáveis } & Md $(\mathrm{Q} 1 ; \mathrm{Q} 3)$ \\
\hline Fatores de motivação & \\
Prevenção de doenças & $5,0(4,5 ; 5,0)$ \\
Condição física & $4,5(3,9 ; 5,0)$ \\
Controle do peso & $3,8(2,8 ; 4,5)$ \\
Aparência & $3,6(2,2 ; 4,3)$ \\
Controle do estresse & $4,8(3,3 ; 5,0)$ \\
Diversão & $4,7(4,0 ; 5,0)$ \\
Afiliação & $5,0(3,0 ; 5,0)$ \\
Reabilitação da saúde & $3,8(3,2 ; 4,70$ \\
Competição & $2,5(1,2 ; 3,4)$ \\
Reconhecimento social & $1,5(0,8 ; 3,1)$ \\
Autoestima & $34,0(32,0 ; 36,0)$ \\
Insatisfação corporal & $-1,0(-2,0 ;-1,0)$ \\
\hline \multicolumn{1}{c}{ Md: mediana. }
\end{tabular}


correlação significativa $(p<0,05)$. Verificou-se que a autoestima apresentou associação significativa $(p<0,05)$ com os fatores de motivação de condição física e diversão, explicando $4 \%$ e $5 \%$ da variabilidade destes motivos, respectivamente (Figura 1).

Em relação às trajetórias individuais do modelo de Path Analysis (Figura 1), verificou-se que a autoestima se associou positivamente com efeito moderado sobre os fatores de condição física $(\boldsymbol{\beta}=0,22)$ e diversão $(\beta=0,24)$, indicando que quanto maior a autoestima da idosa, maior a procura pela prática da hidroginástica para melhorar a condição física e se divertir.

\section{DISCUSSÃO}

Os principais achados desta investigação demonstram que as idosas praticavam a hidroginástica com intuito de prevenir doenças, controlar o estresse e a afiliação e se divertir. Em relação à autoestima, as idosas apresentaram nível satisfatório, enquanto, para a insatisfação corporal apresentaram uma leve insatisfação pela magreza.

O principal achado do estudo foi o modelo de Path (Figura 1), onde os resultados demostraram que a autoestima está diretamente ligada aos motivos para busca da prática da hidroginástica para melhoria do condicionamento físico e diversão. Comprovando assim, que a prática de exercício físico, não se limita a apenas ao bem-estar fisiológico, mas também, o social e o psicológico ${ }^{13}$. Segundo a TAD, o contexto social é algo de suma importância para o desenvolvimento do comportamento autodeterminado e para que o indíviuo consiga sentir prazer e satisfação pela tarefa ${ }^{13}$.

Ao analisar a correlação entre a motivação para a prática da hidroginástica, a autoestima e a insatisfação corporal das idosas, ficou evidente que existe uma associação direta da procura pelo exercício para melhorar a condição física e para se

Tabela 2. Comparação dos fatores de motivação para a prática de hidroginástica das idosas em função do distúrbio de autoestima

\begin{tabular}{lccc}
\hline \multirow{2}{*}{ Fatores de Motivação } & \multicolumn{2}{c}{ Distúrbio de autoestima } & \multirow{2}{*}{ Sim $(\mathrm{n}=8)$} \\
\cline { 2 - 3 } & $\mathrm{Md}(\mathrm{Q} 1-\mathrm{Q} 3)$ & $\mathrm{Não}(\mathrm{n}=62)$ & $\mathrm{Md}(\mathrm{Q} 1-\mathrm{Q} 3)$ \\
\cline { 2 - 3 } & $4,92(4,46-5,00)$ & $5,00(4,50-5,00)$ & 0,760 \\
Prevenção de doenças & $4,13(3,63-5,00)$ & $4,50(3,88-5,00)$ & 0,850 \\
Condição física & $3,50(3,00-5,00)$ & $3,75(2,63-4,31)$ & 0,683 \\
Controle do peso & $3,63(3,06-4,75)$ & $3,63(1,94-4,25)$ & 0,683 \\
Aparência & $4,75(4,00-4,94)$ & $4,75(3,25-5,00)$ & 0,804 \\
Controle do estresse & $4,83(4,04-4,83)$ & $4,58(4,00-5,00)$ & 0,992 \\
Diversão & $5,00(4,31-5,00)$ & $4,88(3,00-5,00)$ & 0,335 \\
Afiliação & $3,83(2,33-4,83)$ & $3,83(3,16-4,66)$ & 0,948 \\
Reabilitação da saúde & $3,70(1,85-4,65)$ & $2,40(1,20-3,20)$ & $0,037^{*}$ \\
Competç̧ão & $2,88(1,63-3,94)$ & $1,50(0,75-2,81)$ & $0,048^{*}$ \\
Reconhecimento social & & &
\end{tabular}

*Diferença significativa $(p<0,05)$ - Teste "U" de Mann-Whitney. Md: mediana. 
Tabela 3. Comparação dos fatores de motivação para a prática de hidroginástica das idosas em função da insatisfação corporal

\begin{tabular}{lccc}
\hline & \multicolumn{2}{c}{ Insatisfação corporal } & \\
\cline { 2 - 3 } Fatores de Motivação & $\begin{array}{c}\text { Sim } \\
(n=56)\end{array}$ & $\begin{array}{c}\text { Não } \\
(n=14)\end{array}$ & $p$ \\
\cline { 2 - 3 } & Md (Q1-Q3) & Md (Q1-Q3) & \\
\cline { 2 - 3 } & $4,50(4,33-5,00)$ & $5,00(5,00-5,00)$ & $0,005^{*}$ \\
Prevenção de doenças & $4,50(4,00-5,00)$ & $4,75(3,38-5,00)$ & 0,635 \\
Condição física & $3,71(2,38-4,44)$ & $3,75(3,69-4,63)$ & 0,341 \\
Controle do peso & $3,38(1,81-4,00)$ & $4,25(3,19-4,81)$ & 0,056 \\
Aparência & $4,75(3,25-5,00)$ & $4,75(4,44-5,00)$ & 0,384 \\
Controle do estresse & $4,58(3,87-5,00)$ & $5,00(4,38-5,00)$ & 0,134 \\
Diversão & $4,50(3,00-5,00)$ & $5,00(4,38-5,00)$ & 0,125 \\
Afiliação & $3,66(2,83-4,46)$ & $4,17(3,00-5,00)$ & 0,471 \\
Reabilitação da saúde & $2,40(1,20-3,35)$ & $2,70(1,85-3,75)$ & 0,436 \\
Competição & $1,50(0,75-2,94)$ & $1,88(0,75-3,50)$ & 0,575 \\
Reconhecimento social & &
\end{tabular}

Tabela 4. Correlação entre os fatores de motivação para a prática da hidroginástica, a autoestima e a insatisfação corporal das idosas

\begin{tabular}{|c|c|c|c|c|c|c|c|c|c|c|c|}
\hline VARIÁVEIS & 2 & 3 & 4 & 5 & 6 & 7 & 8 & 9 & 10 & 11 & 12 \\
\hline 1.Prev. Doenças & $0,41^{*}$ & $0,36^{*}$ & $0,52^{*}$ & $0,49^{*}$ & $0,55^{\star}$ & $0,54^{*}$ & $0,27^{*}$ & $0,24^{*}$ & 0,20 & 0,18 & 0,10 \\
\hline 2.Condição física & & $0,72^{*}$ & $0,68^{*}$ & $0,62^{*}$ & $0,76^{*}$ & $0,64^{*}$ & $0,31^{*}$ & $0,55^{*}$ & $0,52^{*}$ & $0,28^{*}$ & $-0,03$ \\
\hline 3.Controle do peso & & & $0,79^{*}$ & $0,70^{*}$ & $0,69^{*}$ & $0,71^{*}$ & $0,37^{*}$ & $0,51^{*}$ & $0,45^{*}$ & 0,13 & 0,01 \\
\hline 4.Aparência & & & & $0,78^{*}$ & $0,75^{*}$ & $0,81^{*}$ & $0,57^{*}$ & $0,62^{*}$ & $0,55^{\star}$ & 0,15 & 0,02 \\
\hline 5.Cont. Estresse & & & & & $0,74^{*}$ & $0,85^{*}$ & $0,46^{*}$ & $0,59^{*}$ & $0,53^{*}$ & 0,06 & $-0,10$ \\
\hline 6.Diversão & & & & & & $0,79^{*}$ & $0,35^{*}$ & $0,60^{*}$ & $0,54^{*}$ & $0,32^{*}$ & 0,01 \\
\hline 7.Afiliação & & & & & & & $0,46^{*}$ & $0,64^{*}$ & $0,54^{*}$ & 0,07 & $-0,04$ \\
\hline 8.Reab. Saúde & & & & & & & & $0,49^{*}$ & $0,51^{*}$ & 0,09 & $-0,04$ \\
\hline 9.Competição & & & & & & & & & $0,91^{*}$ & $-0,05$ & $-0,10$ \\
\hline 10.Rec. Social & & & & & & & & & & $-0,03$ & $-0,12$ \\
\hline 11.Autoestima & & & & & & & & & & & $0,24^{*}$ \\
\hline 12.Ins. Corporal & & & & & & & & & & & \\
\hline
\end{tabular}

${ }^{*}$ Correlação Significativa $(p<0,05)$ - Correlação de Spearman.

Prev.: prevenção; Cont.: controle; Reab.: reabilitação; Rec.: reconhecimento; Ins: insatisfação.

divertir. Esta questão social abrange os princípios da TAD, na qual compreende o relacionamento social como componente das necessidades psicológicas básicas ${ }^{14}$. Desta forma, um dos motivos dos idosos começarem a praticar exercício é a socialização com outros idosos, tornando o ambiente mais motivador $^{15}$. 
Figura 1. Modelo de Path Analysis da associação da autoestima com os motivos para a prática de hidroginástica das idosas.

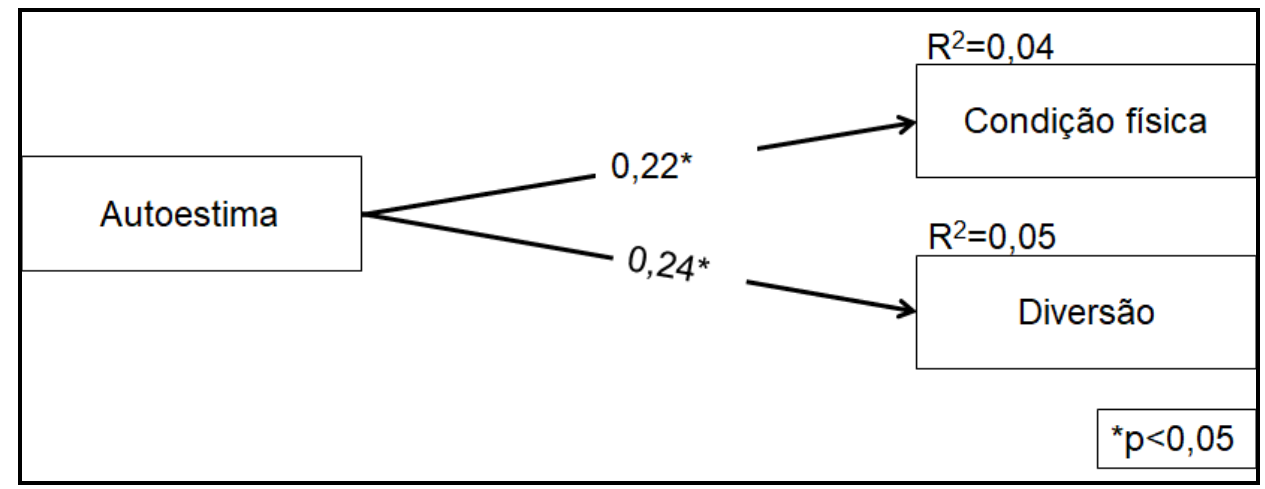

Em mais uma análise, constatou-se que as idosas mais satisfeitas com a sua autoimagem, são mais motivadas a pratica de hidroginástica com intuito de prevenção de doenças, do que as idosas insatisfeitas com o próprio corpo. A saúde é o principal motivo para os idosos realizarem atividade física ${ }^{16}$. Embora, os achados evidenciem que as idosas se preocupavam com a aparência, Silva et al. ${ }^{17}$ observaram que a preocupação com a autoimagem é algo que diminui com o envelhecimento, uma vez que os cuidados se voltam para o bem-estar e a saúde.

Quando comparamos fatores de motivação para a prática de hidroginástica das idosas em função do distúrbio de autoestima, verificou-se que as idosas, com presença de distúrbio de autoestima, são mais motivadas para a prática da hidroginástica para competir e para serem reconhecidas socialmente do que as idosas com ausência de distúrbio de autoestima. Esses resultados não vão de encontro com a literatura, onde foi demostrado que o fator competitividade foi o menos importante para os idosos ${ }^{17}$, os quais ressaltam que o idoso não procura a atividade física para competir e sim, para cuidar da saúde e se socializar.

Por fim, os achados estão de acordo com a literatura em estudos anteriores, relacionados aos motivos para o idoso se exercitar ${ }^{1,17}$. Porém, este estudo apresenta algumas limitações que merecem ser destacadas: trata-se de uma pesquisa transversal, o que impede inferir a causalidade; a amostra é relativamente pequena e engloba apenas idosos do sexo feminino, de um único município do noroeste paranaense, o que impede generalizar os dados apresentados.

\section{CONCLUSÃO}

A partir dos resultados obtidos, podese concluir que a autoestima e a insatisfação corporal são fatores intervenientes na motivação de idosas para a prática de atividade física. Especificamente, o distúrbio de autoestima parece estar associado à 
busca pelo reconhecimento social na prática da atividade física e a satisfação corporal leva as idosas a procurar a atividade física apenas para prevenir doenças. Além disso, a autoestima pode favorecer a busca pela atividade física para melhorar a saúde física e para a diversão. Do ponto de vista prático,

\section{REFERÊNCIAS}

1. Gutiérrez M, Calatayud P, Tomás JM. Motives to practice exercise in old age and successful aging: A latent class analysis. Archives of gerontology and geriatrics. 2018;77(1):44-50.

2. Franco MR, Tong A, Howard K, Sherrington C, Ferreira PH, Pinto RZ, et al. Older people's perspectives on participation in physical activity: a systematic review and thematic synthesis of qualitative literature. British J Sports Medicine. 2015; 49(19):1268-1276.

3. Copatti SL, Kuczmainski AG, Sá CA, Ferretti F. Imagem corporal e autoestima em idosos: uma revisão integrativa da literatura. Estudos Interdisciplinares sobre o Envelhecimento. 2017;22(3):47-62.

4. Pereira ÉF, Teixeira CS, Borgatto AF, Daronco LSE. Relação entre diferentes indicadores antropométricos ea percepção da imagem corporal em idosas ativas. Arch Clinical Psychiatry. 2009; 36(2):54-59.

5. Gomes HM, Oliveira LB, Boas DTV, Lucena ALR, Vieira KFL, Freitas FFQF. Satisfação com imagem corporal: perspectiva de idosos inseridos em um grupo de convivência. Rev de Ciências da Saúde Nova Esperança. 2014;12(2):71-79.

6. Biedenweg K, Meischke H, Bohl A, Hammerback K, Williams $\mathrm{B}$, Poe $\mathrm{P}$, et al. Understanding older adults' motivators and barriers to participating in organized programs supporting exercise behaviors. The Journal of Primary Prevention. 2014;35(1):1-11.

7. Gomes Júnior FF, Brandão AB, Almeida FJM, Oliveira JGD. Compreensão de Idosos sobre os Benefícios da Atividade Física. Ver Bras de Ciências da Saúde. 2016;19(3):193-198. destaca-se a importância do exercício físico regular para o idoso manter níveis satisfatórios de autoestima e satisfação corporal, uma vez que estas condições favorecem a permanência do idoso no exercício físico por motivos de saúde.

8. Agostini CM, Rodrigues VS, Guimarães AC, Damázio LCM, Vasconcelos NN. Análise do desempenho motor e do equilíbrio corporal de idosos ativos com hipertensão arterial e diabetes tipo 2. Rev de Atenção à Saúde. 2018;16(55):29-35.

9. Folstein MF, Folstein SE, McHugh PR. "Mini-mental state" A practical method for grading the cognitive state of patients for the clinician. J Psychiatric. 1975; 2(3):189-198.

10. Brucki SMD, Nitrini R, Caramelli P, Bertolucci PHF, Okamoto $\mathrm{IH}$. Sugestões para o uso do mini-exame do estado mental no Brasil. Arquivos de Neuropsiquiatria. 2003; 61(3B):777-781.

11. Bavoso D, Galeote L, Montiel JM, Cecato JF. Motivação e autoestima relacionada à prática de atividade física em adultos e idosos. Rev Bras de Psicologia do Esporte. 2018;7(2):26-37.

12. Stunkard AJ, Sorenson T, Schulsinger F. Use of the Danish Adoption Register for the study of obesity and thinness. The genetics of neurological and psychiatric disorders. New York: Raven Press; 1983. p.115-20.

13. Chung PK, Zhao Y, Liu JD, Quach B. A canonical correlation analysis on the relationship between functional fitness and health-related quality of life in older adults. Arch of gerontology and geriatrics. 2017; 68(1):44-48.

14. Carvalho R, Pires CMR, Junqueira GC, Freitas D, MarchiAlves LM. Magnitude e duração da resposta hipotensora em hipertensos: exercício contínuo e intervalado. Arq Bras de Cardiologia. 2015;104(3):234-241.

15. Castro MR, Lima LHR, Duarte ER. Jogos recreativos para a terceira; idade: uma análise a partir da percepção dos idosos. Rev Bras de Ciências do Esporte. 2016; 38(3):283-289. 
16. Cardoso AMN. Fatores Motivacionais de Idosos Associados à Prática Regular de Exercícios Físicos. 122f (Dissertação de Mestrado, Instituto de Biociências, Universidade Estadual Paulista, Rio Claro, SP), 2014.

17. Silva ANC, Castanho GKF, Chiminazzo JGC, Barreira J, Fernandes PT. Fatores motivacionais relacionados à prática de atividades físicas em idosos. Psicologia em Estudo. 2016; 21(4): 677-685.

\section{CORRESPONDÊNCIA}

Daniel Vicentini de Oliveira

Avenida Londrina, 934. Apto 1907, torre A.

CEP: 87050-730. Maringá/PR

E-mail: d.vicentini@hotmail.com 